\title{
Erratum to: Nudging museums attendance: a field experiment with high school teens
}

\author{
Patrizia Lattarulo $^{1}$ - Marco Mariani ${ }^{1}$. \\ Laura Razzolini ${ }^{2}$ (D)
}

\section{Erratum to: J Cult Econ \\ DOI 10.1007/s10824-016-9285-6}

In the original article, the Tables 1, 2 and 4 are published incorrectly. The correct tables are given below. The original article was corrected.

The online version of the original article can be found under doi:10.1007/s10824-016-9285-6.

\section{Laura Razzolini}

lrazzolini@vcu.edu

Patrizia Lattarulo

patrizia.lattarulo@irpet.it

Marco Mariani

marco.mariani@irpet.it

1 IRPET - Tuscany's Regional Institute for Economic Planning, Villa La Quiete alle Montalve, Via Pietro Dazzi, 1, 50141 Firenze, Italy

2 Department of Economics, School of Business, Virginia Commonwealth University, 301 West Main Street, Box 4000, Richmond, VA 23284, USA 
Table 1 Experimental design and descriptive statistics about students

\begin{tabular}{|c|c|c|c|c|}
\hline & \multicolumn{4}{|l|}{ Treatment } \\
\hline & Overall & Flier & Present & Reward \\
\hline \multicolumn{5}{|l|}{ Information about the sample } \\
\hline No. of classes & 15 & 5 & 5 & 5 \\
\hline No. of Students $(\%)$ & $294(100 \%)$ & $93(32 \%)$ & $97(33 \%)$ & $104(35 \%)$ \\
\hline \multicolumn{5}{|l|}{ Information about the students } \\
\hline Male $(1 / 0)$ & 0.354 & 0.215 & 0.299 & 0.529 \\
\hline Born abroad (1/0) & 0.061 & 0.022 & 0.093 & 0.067 \\
\hline Suburban resident(1/0) & 0.422 & 0.387 & 0.320 & 0.548 \\
\hline Older than cohort $(1 / 0)$ & 0.058 & 0.075 & 0.062 & 0.039 \\
\hline \multicolumn{5}{|l|}{ Information about the high school } \\
\hline Most friends are classmates $(1 / 0)$ & 0.486 & 0.452 & 0.474 & 0.529 \\
\hline No. classmates who are friends & 3.582 & 4.441 & 2.635 & 3.773 \\
\hline Current GPA (out of 10) & 6.817 & 6.720 & 6.789 & 6.931 \\
\hline Interested only in humanities $(1 / 0)$ & 0.262 & 0.226 & 0.392 & 0.173 \\
\hline \multicolumn{5}{|l|}{ Leisure and Cultural habits } \\
\hline Interested in politics $(1 / 0)$ & 0.228 & 0.215 & 0.206 & 0.260 \\
\hline Volunteer $(1 / 0)$ & 0.167 & 0.204 & 0.144 & 0.154 \\
\hline No. museum visits during last year & 3.867 & 3.269 & 4.742 & 3.587 \\
\hline Visited Palazzo Vecchio previously (1/0) & 0.721 & 0.677 & 0.763 & 0.721 \\
\hline \multicolumn{5}{|l|}{ Parents' education } \\
\hline At least one parent unemployed (1/0) & 0.157 & 0.151 & 0.175 & 0.144 \\
\hline Both parents high school degree (1/0) & 0.327 & 0.376 & 0.247 & 0.356 \\
\hline At least one has college degree (1/0) & 0.455 & 0.398 & 0.516 & 0.452 \\
\hline Parents regularly go to museums $(1 / 0)$ & 0.197 & 0.183 & 0.247 & 0.163 \\
\hline
\end{tabular}


Table 2 Timeline

\begin{tabular}{|c|c|}
\hline When & $\mathrm{W}=$ flier $\mathrm{W}=$ presentation $\mathrm{W}=$ reward \\
\hline $\begin{array}{l}\text { First visit } \\
\text { Late March/early April } \\
2014\end{array}$ & $\begin{array}{l}\text { Students are informed that they will be involved in a study about cultural } \\
\text { consumption. A flier of Palazzo Vecchio with opening hours is } \\
\text { distributed. A brief text written by the experimenters stating the } \\
\text { importance of museums' attendance is also distributed } \\
\text { A museum operator talks about Palazzo Vecchio for } 15 \text { min } \\
\text { Students are told that the visit to Palazzo } \\
\text { Vecchio will be rewarded with extra } \\
\text { credit points }\end{array}$ \\
\hline & $\begin{array}{l}\text { Students complete a questionnaire about their background characteristics, } \\
\text { cultural consumption habits and within-classroom friendship ties } \\
\text { Students who visit Palazzo Vecchio within two months bring the entry } \\
\text { ticket back to their teacher }\end{array}$ \\
\hline $\begin{array}{l}\text { Second visit } \\
\text { After two months }\end{array}$ & Collect entry tickets from the assigned teachers \\
\hline $\begin{array}{l}\text { Third visit } \\
\text { After eight months (six } \\
\text { months after collection of } \\
\text { entry ticket) }\end{array}$ & $\begin{array}{l}\text { Students complete a questionnaire about the number of individual visits to } \\
\text { museums done in town and out of town in the past six months }\end{array}$ \\
\hline
\end{tabular}

Table 4 Descriptive statistics at the class level by treatment

\begin{tabular}{|c|c|c|c|c|c|c|}
\hline Variable & Obs & Mean & SD & Min & Median & $\operatorname{Max}$ \\
\hline \multicolumn{7}{|l|}{ Flier } \\
\hline $\begin{array}{l}\text { (a) share of students undertaking the encouraged } \\
\text { visit }\end{array}$ & 5 & 0.033 & 0.075 & 0.000 & 0 & 0.167 \\
\hline (b) No. of voluntary museum visits six months later & 5 & 1.756 & 2.013 & 0.000 & 1.158 & 4.875 \\
\hline $\begin{array}{l}\text { (c) No. of voluntary museum visits in previous } \\
12 \text { months }\end{array}$ & 5 & 3.286 & 0.677 & 2.760 & 3.158 & 4.444 \\
\hline Difference $(b-c)$ & 5 & -1.530 & 1.541 & -3.222 & -2.000 & 0.431 \\
\hline \multicolumn{7}{|l|}{ Presentation } \\
\hline $\begin{array}{l}\text { (a) share of students undertaking the encouraged } \\
\text { visit }\end{array}$ & 5 & 0.118 & 0.263 & 0.000 & 0 & 0.588 \\
\hline (b) No. of voluntary museum visits six months later & 5 & 4.303 & 2.346 & 2.500 & 3.263 & 8.368 \\
\hline $\begin{array}{l}\text { (c) No. of voluntary museum visits in previous } \\
12 \text { months }\end{array}$ & 5 & 4.749 & 1.237 & 3.760 & 4.533 & 6.857 \\
\hline Difference $(b-c)$ & 5 & -0.446 & 1.303 & -2.147 & -0.522 & 1.511 \\
\hline \multicolumn{7}{|l|}{ Reward } \\
\hline $\begin{array}{l}\text { (a) share of students undertaking the encouraged } \\
\text { visit }\end{array}$ & 5 & 0.402 & 0.180 & 0.222 & 0.455 & 0.650 \\
\hline (b) No. of voluntary museum visits six months later & 5 & 2.980 & 0.622 & 2.190 & 2.909 & 3.909 \\
\hline $\begin{array}{l}\text { (c) No. of voluntary museum visits in previous } \\
12 \text { months }\end{array}$ & 5 & 3.543 & 1.058 & 2.667 & 3.050 & 5.000 \\
\hline Difference $(b-c)$ & 5 & -0.564 & 0.679 & -1.409 & -0.491 & 0.111 \\
\hline
\end{tabular}

